\author{
Nicola Morelli \\ Sara Gori \\ Gianfranco Cafforio \\ Simone Gallerini \\ Filippo Baldacci \\ Giovanni Orlandi \\ Luigi Murri
}

\section{Prevalence of right-to-left shunt in patients with cluster headache}

Published online: 20 July 2005

N. Morelli $(\bowtie) \cdot S$. Gori • G. Cafforio

S. Gallerini $•$ F. Baldacci

G. Orlandi • L. Murri

Department of Neurosciences,

Institute of Neurology,

University of Pisa,

Via Roma 67, I-56125 Pisa, Italy

e-mail: n.morelli@inwind.it

Tel.: +39-050-992851/992443

Fax: +39-050-554808

\begin{abstract}
Recent investigations documented that the prevalence of right-to-left shunt (RLS) in patients with migraine with aura (MA) is significantly higher than in healthy controls and similar to prevalence of RLS in young patients with cryptogenic stroke (CS). Nevertheless, little data are available in the literature about RLS prevalence in the other forms of primary headache. The aim of this study was to investigate the occurrence of RLS in patients with cluster headache $(\mathrm{CH})$. We enrolled 30 consecutive patients with $\mathrm{CH}$ diagnosis according to the IHS cri-
\end{abstract}

teria and 40 controls. RLS was assessed with bilateral transcranial Doppler contrast (TCDc) monitoring of middle cerebral arteries. Eleven patients $(37 \%)$ resulted positive to TCDc monitoring for evaluation of RLS. These data show that the presence of RLS in this group is more prevalent than in the general population and similar to that found in MA and in CS.

Key words Cluster headache • Right-to-left shunt $\cdot$ Patent foramen ovale $\cdot$ Transcranial Doppler

\section{Introduction}

The prevalence of right-to-left shunt (RLS) has been documented recently to be significantly higher in patients suffering from migraine with aura (MA) than in healthy controls and similar to that found in young patients with stroke [1-3]. These results suggest the possible role of RLS in increased risk of stroke in migraineurs and in the pathophysiology of headache. It has been hypothesised for MA that the relationship with RLS may be genetically determined [4]. Nevertheless little data are available in the literature about RLS in the other forms of primary headache [5]. This study was designed to evaluate the prevalence of RLS in subjects with cluster headache $(\mathrm{CH})$ and to compare it with the prevalence of RLS in a control group.

\section{Material and methods}

We enrolled 30 (22 females and 8 males, mean age $36.4 \pm 8.2$ years, range 26-51 years) consecutive patients with $\mathrm{CH}$ diagnosis according to the IHS criteria [6] evaluated at the Headache Center of the Neurosciences Department, University of Pisa. We also evaluated 40 control subject, matched for age and sex, without history of $\mathrm{CH}$ or other primary headache. All patients gave their written informed consent before being admitted to the study.

RLS was assessed with bilateral transcranial Doppler contrast (TCDc) (DWL Multidop X4-TCD 7, FRG) monitoring of both middle cerebral arteries during normal ventilation, and by Valsalva manoeuvres according to the Consensus Meeting of the European Society of Neurosonology [7]. An agitated saline solution $(9 \mathrm{ml})$ mixed with $1 \mathrm{ml}$ of air was injected into an antecubital vein and RLS was diagnosed if at least one MB was record- 
ed within $15 \mathrm{~s}$ of the injection. RLS was classified as latent if the MB was documented only during the Valsalva manoeuvre and as permanent if the MB was detected during normal ventilation as well. With regard to the degree of the RLS, this was classified as low grade (1-10 MBs), medium grade (11-20 MBs) and highgrade ( $>20$ MBs or curtain effect). Transoesophageal echocardiography was subsequently performed to confirm the presence of patent foramen ovale in all patients.

No patient assumed pharmacological prophylaxis for $\mathrm{CH}$ or any other pharmacological treatment in the three months before enrolment; none of them had a history of internal medicine or neuropsychiatric pathologies or any abnormalities detected on cranial CT scan and/or MRI, and carotid stenosis was documented to be absent by Duplex scanner. The statistical analysis was performed using the $\chi^{2}$ test.

\section{Results}

The subjects with $\mathrm{CH}$ had a mean age of $37 \pm 12.3$ years and a male/female ratio of $22 / 8$. The controls had a mean age of $42 \pm 11.3$ years and a male/female ratio of $22 / 8$. The episodic form of $\mathrm{CH}$ was present in 27 patients. The remaining 3 patients had the chronic form. Eleven patients $(37 \%)$ resulted positive to TCD contrast monitoring for evaluation of RLS and were divided in two groups: the first one with latent RLS, the second group with permanent RLS. In the first group 8 patients (72\%) showed a low-grade RLS. In the second group 3 patients (28\%) showed a high-grade RLS. Transoesophageal echocardiography confirmed the presence of PFO in all the cases.

A curtain effect was present in one of the patients with permanent high-grade RLS. A RLS was found in $18.3 \%$ of control subjects. The difference of prevalence of RLS between $\mathrm{CH}$ patients and controls was significant $(p<0.005)$. No significant differences have been detected between the characteristics of the shunt in the two groups.

\section{Discussion}

Our study show that RLS is present in $37 \%$ of patients suffering from $\mathrm{CH}$, and it is the second investigation aimed at assessing the prevalence of RLS in $\mathrm{CH}$ patients revealed by the number of MBs detected during TCDc monitoring. This prevalence is similar to that found in a recent Italian study (42.5\%) [5] and in other studies involving MA and cryptogenic stroke (CS) patients [1-3], so confirming that in another kind of primary headache the prevalence of RLS is higher than in the general population.

Pathogenetic speculations on a possible correlation between patients with patent foramen ovale and headache, particularly in MA, have sprouted from therapeutic successes producing improvement of the clinical picture after closure of patent foramen ovale [8-12].

It has been suggested that RLS plays a role in the aura attack onset in some patients, probably by a mechanism of paradoxical embolism of platelet aggregates or other chemicals. Moreover it has been hypothesised that for MA and $\mathrm{CH}$ the relationship with RLS shunt may be genetically determined [5]. A report documented that the occurrence of atrial shunts was consistent with autosomal dominant inheritance in some families with MA [4]. Leone et al. [13] suggested that $\mathrm{CH}$ could be, at least in part, of genetic origin. In a previous study we did not find any relationship between the extent of the RLS and severity of headache. It is probably caused by the absence of a pathogenic link between these conditions [14]. It is possible that a particular genetical substrate can determinate endocardial abnormalities and a predisposition to headache (migraine or $\mathrm{CH}$ ) [3-5].

\section{References}

1. Del Sette M, Angeli S, Leandri M, Ferriero G, Bruzzone GL, Finocchi C, Gandolfo C (1998) Migraine with aura and right-to-left shunt on transcranial Doppler: a case-control study. Cerebrovasc Dis 8:327-330

2. Anzola GP, Magoni M, Guindani M, Rozzini L, Dalla Volta G (1999) Potential source of cerebral embolism in migraine with aura: a transcranial Doppler study. Neurology 53:2213-2214
3. Sztajzel R, Genoud D, Roth S, Mermillod B, Le Floch-Rohr J (2002) Patent foramen ovale, a possible cause of symptomatic migraine: a study of 74 patients with acute ischemic stroke. Cerebrovasc Dis 13:102-106

4. Wilmshurst PT, Pearson MJ, Nightingale S, Walsh KP, Morrison WL (2004) Inheritance of persistent foramen ovale and atrial septal defects and the relation to familial migraine with aura. Heart 90:1315-1320
5. Finocchi C, Del Sette M, Angeli S, Rizzi D, Gandolfo C (2004) Cluster headache and right-to-left shunt on contrast transcranial Doppler: a casecontrol study. Neurology 63:1309-1310

6. Headache Classification Subcommittee of the International Headache Society (2004) Classification and diagnostic criteria for headache disorders, cranial neuralgias and facial pain, 2nd edn. Cephalalgia [Suppl 1]:1-160 
7. Angeli S, Del Sette M, Beelke M, Anzola GP, Zanette E (2001)

Transcranial Doppler in the diagnosis of cardiac patent foramen ovale. Neurol Sci 22:353-356

8. Wilmshurst PT, Nightingale $S$, Walsh KP (2000) Effect on migraine of closure cardiac right-to-left shunts to prevent recurrence of decompression illness or stroke or for haemodynamic reasons. Lancet 356:1648-1651

9. Morandi E, Anzola GP, Angeli S, Melzi G, Onorato E (2003)

Transcatheter closure of patent foramen ovale: a new migraine treatment? J Interv Cardiol 16:39-42
10. Post MC, Thijs V, Herroelen L, Budts WI (2004) Closure of a patent foramen ovale is associated with a decrease in prevalence of migraine. Neurology 62:1439-1440

11. Schwerzmann M, Wiher S, Nedeltchev K, Mattle HP, Wahl A, Seiler C, Meier B, Windecker S (2004) Percutaneous closure of patent foramen ovale reduces the frequency of migraine attacks. Neurology 62:1399-1401

12. Welch KMA (2003) Stroke and migraine-the spectrum of cause and effect. Funct Neurol 18:121-126
13. Leone M, Russell MB, Rigamonti A, Attanasio A, Grazzi L, D' Amico D, Usai S, Bussone G (2001) Increased familial risk of cluster headache. Neurology 56:1233-1236

14. Gori S, Morelli N, Fanucchi S, Orlandi G, Murri L (2004) Cerebrovascular disease and migraine: pathogenetic implications and therapeutics opportunities. J Headache Pain 5[Suppl 1]:S24 (Abstract) 